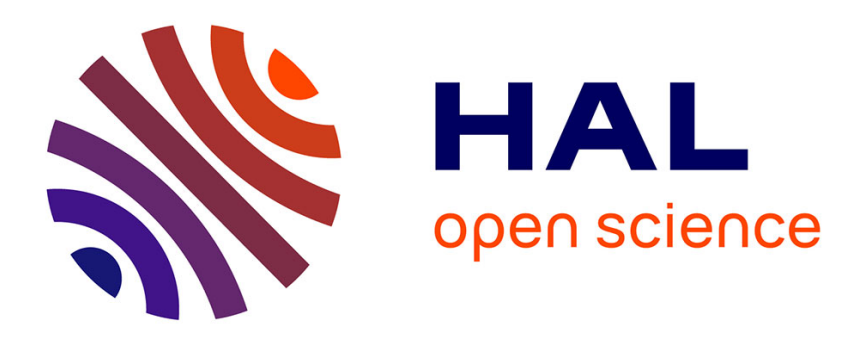

\title{
La compétition mondiale de l'intelligence artificielle
}

Charles Thibout

\section{To cite this version:}

Charles Thibout. La compétition mondiale de l'intelligence artificielle. Pouvoirs - Revue française d'études constitutionnelles et politiques, 2019, 170 (3), pp.131-142. 10.3917/pouv.170.0131 . hal03031260v2

\section{HAL Id: hal-03031260 \\ https://hal.science/hal-03031260v2}

Submitted on 1 May 2021

HAL is a multi-disciplinary open access archive for the deposit and dissemination of scientific research documents, whether they are published or not. The documents may come from teaching and research institutions in France or abroad, or from public or private research centers.
L'archive ouverte pluridisciplinaire HAL, est destinée au dépôt et à la diffusion de documents scientifiques de niveau recherche, publiés ou non, émanant des établissements d'enseignement et de recherche français ou étrangers, des laboratoires publics ou privés. 


\title{
LA COMPÉTITION MONDIALE DE L'INTELLIGENCE ARTIFICIELLE
}

\author{
Charles Thibout
}

\section{Résumé}

Les perspectives ouvertes par l'intelligence artificielle, en particulier sur son versant économique et militaire, ont rejoint les ambitions internationales de certains pays et précipité la naissance d'une nouvelle compétition. L'antagonisme sino-américain se structure désormais autour de ce répertoire de techniques, suivant des stratégies distinctes : un modèle dirigiste, fondé sur un complexe techno-partidaire, côté chinois, face au modèle néolibéral américain, qui institue la primauté et l'autonomie d'action des grandes firmes numériques.

Un sentiment diffus imprime inexorablement sa marque, inspirant partout crainte et convoitise dans l'esprit des hommes. Le $1^{\text {er }}$ septembre 2017, à Iaroslavl, Vladimir Poutine le traduisait en ces termes : "Celui qui deviendra leader [en intelligence artificielle] sera le maître du monde. » La conduite des grandes puissances accentue encore ce sentiment : chacun expose son «plan stratégique » de développement de l'intelligence artificielle, comme si ce répertoire de techniques renfermait le secret de la puissance, et peut-être même de la suprématie. Après les travaux précurseurs d'Alan Turing, au tournant des années 1950, les scientifiques américains s'imposèrent dans le champ de l'IA, soutenus par le Pentagone, avant que ne débutât une première forme de compétition avec les Britanniques et les Japonais au milieu des années 1980, qui fit long feu. Les espoirs déçus de l'IA ne devaient renaître qu'à 
l'aube des années 2010, par la conjonction des progrès techniques et de la remise en cause de l'hégémonie américaine, jusqu'à fournir l'épitase des tensions interétatiques.

La course à l'intelligence artificielle institue une véritable compétition à plus d'un titre : les principaux rivaux, États-Unis et Chine en tête, s'accordent (competere) à dire que ces nouvelles technologies, les matières premières nécessaires à leur élaboration, revêtent un caractère stratégique et, partant, estiment que celui qui en maîtrisera le plus haut niveau de sophistication subjuguera fatalement tous les autres. Les efforts déployés, dans tous les domaines de la vie sociopolitique et internationale, pour s'adjuger des technologies si ondoyantes, si mal comprises par les décideurs politiques, procèdent d'une vision fantasmatique de l'intelligence artificielle, quant aux possibles qu'elle semble ouvrir et quant aux situations culturelles et géopolitiques rencontrées avec lesquelles elle entre en congruence. L'eschatologie chinoise d'un retour à la grandeur impériale, cent ans après l'avènement de la République populaire, et le messianisme techniciste d'une Amérique inquiète de son avenir sont deux puissants moteurs de croyance en la promesse apothéotique ou salvatrice - c'est selon - de l'intelligence artificielle. Cette rivalité est indissociable d'un contexte international en profonde mutation : le mythe de l'hyperpuissance américaine égare toujours plus de fidèles; la Chine et la Russie mettent tout en œuvre pour reconquérir et asseoir leur statut de grande puissance. Aussi l'intelligence artificielle apparaît-elle opportunément dans l'arène internationale comme un accélérateur instrumental des antagonismes interétatiques, au même titre, d'une certaine manière, que l'arme nucléaire, à cette différence près que ce nouvel ensemble technologique paraît à même d'établir la supériorité d'un acteur dans tous les domaines de l'activité humaine. Du moins est-ce ainsi qu'on le présente.

Toutefois, cette compétition entre puissances n'est pas exclusive d'autres types d'acteurs. Des puissances secondaires, mais, surtout, des acteurs non étatiques tels que les firmes transnationales numériques, y jouent un rôle ambivalent et pour le moins significatif. Les grandes entreprises du Web américaines (les «GAFAM ${ }^{1}$ ) concentrent les investissements, les innovations, les technologies les plus avancées et les meilleurs ingénieurs du secteur. Leur

1. Pour Google (Alphabet), Amazon, Facebook, Apple, Microsoft, ainsi que des entreprises comme Cisco, IBM, Intel, Oracle et quelques autres. 
puissance technique incomparable place dans leur dépendance les États qui aspirent à coiffer leurs concurrents, en tant que ces États se sont résolus, plus ou moins activement, à déléguer leurs compétences et leur base industrielles et technologiques à la sphère privée, domestique puis étrangère. Désormais, pour nombre d'États, ce que l'on appelle les technologies émergentes, au premier rang desquelles l'intelligence artificielle, ne sont accessibles que par l'accord exprès d'entreprises qui les maîtrisent complètement, depuis la conception jusqu'à la livraison. Cette situation autorise les firmes numériques à faire effraction dans le champ des relations internationales aux côtés des États-gladiateurs hobbesiens : le stato-centrisme de la théorie réaliste semble dès lors moins apte à rendre compte de la complexité de la course à l'intelligence artificielle qu'une perspective transnationaliste.

\section{Les ressorts de la compétition interétatique}

Le mode traditionnel d'interprétation de la compétition pour l'intelligence artificielle comme phénomène essentiellement interétatique est légitime. Il s'appuie sur les réminiscences, fussent-elles anachroniques, de l'opposition binaire de la guerre froide, mais aussi sur la personnification de cet antagonisme sous les traits de Donald Trump et de Xi Jinping. Plus radicalement, cette compétition s'origine dans une volonté étatique d'appropriation et d'instrumentalisation de ces technologies qui remonte à la Seconde Guerre mondiale.

\section{De la cybernétique aux premières interventions étatiques}

Très tôt dans son histoire, l'intelligence artificielle a eu partie liée avec les mondes académique et militaire, notamment aux États-Unis. Le mouvement cybernétique des années 1940 et 1950, dont sont issus les principaux schèmes scientifiques de l'IA, fut animé par une constellation de savants gravitant autour de l'appareil de défense américain. Le plus célèbre d'entre eux, le mathématicien Norbert Wiener, travailla au sein du National Defense Research Committee (ancêtre de l'ARPA et de la DARPA), dès 1940, où il tenta de concevoir un appareil permettant le tir prédictif antiaérien. Ce projet, «AA Predictor », sera mené conjointement avec Julian Bigelow, autre figure du mouvement cybernétique, et décrit dans un document 
ultérieur, déclassifié en 1949 et surnommé le Yellow Peril. Dix des vingt-quatre principaux membres du premier mouvement cybernétique furent ainsi directement impliqués dans des activités scientifiques à visée militaire aux États-Unis, dont le projet Manhattan. Ce sont tout spécialement les notions de prédiction, d'aide à la prise de décision, d'homologie et d'interaction entre l'homme et la machine qui intéressaient alors le Pentagone et qui seraient au centre des recherches en IA dans les décennies suivantes.

Après que l'intelligence artificielle eut été fondée en champ de recherche autonome, en 1956, par le cybernéticien John McCarthy, le département de la Défense américain comprit rapidement l'avantage qu'il pourrait retirer des technologies issues de cette discipline. En 1962, l'Advanced Research Projects Agency (ARPA), par l'entremise de son Information Processing Techniques Office (IPTO), commença à financer la recherche en intelligence artificielle du groupe dirigé par Marvin Minsky au MIT. Le premier contrat, d'une valeur de 2,2 millions de dollars, fut renouvelé chaque année pendant plus d'une décennie et élargi dès 1963 aux programmes de recherche en IA de Stanford, supervisé par John McCarthy, et de l'université Carnegie-Mellon ${ }^{2}$. Néanmoins, le manque de résultats mit pratiquement fin aux financements au milieu des années 1970, avant de reprendre face à la recrudescence des recherches menées autour des «systèmes experts » et, surtout, après que le gouvernement japonais eut lancé, en 1982, son projet de «Fifth Generation Computer Systems » financé au départ à hauteur de 850 millions de dollars et censé relancer les espoirs dans l'IA. De crainte que les Japonais ne réalisent ce qu'ils n'avaient su faire auparavant, les Américains et les Européens financèrent de nouveaux projets de recherche: la Defense Advanced Research Projects Agency (DARPA) mit en œuvre une Strategic Computing Initiative avec un budget total d'un milliard de dollars ${ }^{3}$; les Européens lancèrent le programme ESPRIT, et les Britanniques le programme Alvey... Mais, derechef, les espoirs furent déçus. Les Américains réussirent néanmoins à s’imposer dans les années 1990, aidés par les succès d'IBM (Deep Blue) et les financements continus de la DARPA.

2. Cf. l'interview de Joseph Carl Robnett Licklider, premier directeur de l'IPTO, in Thomas C. Bartee (dir.), Expert Systems and Artificial Intelligence, Indianapolis (Ind.), Howard W. Sams \& Company, 1988, p. 213-290.

3. Alex Roland, avec Philip Shiman, Strategic Computing: DARPA and the Quest for Machine Intelligence, 1983-1993, Cambridge (Mass.), MIT Press, 2002. 
Dans le contexte post-guerre froide, où la menace représentée par les autres États semblait peu signifiante, l'intelligence artificielle, éclipsée par le terrorisme, n'était plus une priorité. Seules les agences de renseignement américaines continuaient d'y voir un ensemble technologique d'avenir.

\section{Affronter la menace russe et chinoise}

Au début des années 2010, la menace terroriste semblait s'éloigner, cependant que la Russie et la Chine affichaient de plus en plus leurs ambitions internationales et leur maîtrise des nouvelles technologies. En 2014, la crise ukrainienne permit de mesurer l'art russe dans la conduite de la guerre hybride, en particulier dans la cyberguerre et la guerre informationnelle. En décembre 2013, la Chine fut le premier pays à faire alunir un engin spatial depuis 1976. Surtout, depuis la fin des années 1990, Pékin affichait son ambition de contrecarrer la puissance américaine par le truchement de la guerre électronique. En 1999, deux colonels de l'armée de l'air, Qiao Liang et Wang Xiangsui, avaient proposé une stratégie d'affrontement de la puissance américaine, par une manière de guerre asymétrique, dérégulée, où l'outil cyber devait permettre de compenser la prépondérance militaire des États-Unis ${ }^{4}$. Depuis lors, cette stratégie a été adoptée par l'Armée populaire de libération, qui mise sur son rattrapage technologique et sa maîtrise des outils numériques pour contrarier l'hégémonie américaine et élever la Chine au rang de puissance internationale - et même de puissance dominante, à l'horizon 2049, cent ans après l'avènement de la République populaire.

Les États-Unis pressentent le danger qui pèse sur leur prééminence : ils craignent que la Russie et, surtout, la Chine substituent leur supériorité techno-militaire à la leur, celle-là même qui leur permit d'asseoir progressivement leur hégémonie à partir de la Seconde Guerre mondiale. C'est la raison pour laquelle ils lancèrent, en 2014, la Third Offset Strategy, une stratégie de «compensation » de la montée en puissance russe et chinoise, centrée sur les technologies émergentes issues du secteur civil, parmi lesquelles l'intelligence artificielle tient une place de premier choix. Il est notable que les deux premières Offset Strategies avaient été établies dans les années 1950 et 1970 pour contrer la menace soviétique : la reprise de ce

4. La Guerre hors limites (1999), Paris, Payot \& Rivages, 2003. 
concept, dans les années 2010, traduit l'apparition d'une blessure narcissique collective, d'un profond vacillement du mythe de l'hyperpuissance américaine au sein même des instances dirigeantes du pays et, en particulier, du Pentagone. La place centrale qu'y occupe l'intelligence artificielle doit, certes, beaucoup aux progrès impressionnants réalisés depuis 2012, notamment par la technique du deep learning, mais surtout à l'influence exercée par ce que le politiste Stanley Hoffmann appelait la «pensée experte » aux États-Unis : la valeur extraordinaire accordée à la technologie dans le monde politique et social américain comme outil de résolution des problèmes publics ${ }^{5}$.

\section{L'entrée de la Chine dans la course à l'intelligence artificielle, le reste du} monde en retrait

Dans les premières années qui suivirent le lancement de la Third Offset Strategy, la Chine n'accorda qu'une valeur marginale à l'intelligence artificielle, d'autant que les annonces américaines ne furent en réalité guère suivies d'effets. Cependant, l'attitude de la Chine changea radicalement en 2016, lorsqu'une machine conçue par DeepMind (AlphaGo), la filiale britannique de Google, triompha de l'un des tout meilleurs joueurs de go au monde, le Coréen Lee Sedol. Dès lors, les instances dirigeantes du Parti communiste, ainsi que toute l'administration civile et militaire, s'emparèrent de cette question, multiplièrent les colloques et les séminaires sur le sujet, développèrent la notion $d^{\prime}$ " intelligenciation » de la guerre ${ }^{6}$, et commencèrent à établir un plan d'action pour rivaliser avec ce qui apparaissait comme le signe d'un raffermissement de la prévalence technologique américaine. En juillet 2017, le Conseil des affaires de l'État chinois publia un «plan de développement de la prochaine génération d'intelligence artificielle », auquel fut alloué un budget de 22 milliards de dollars, qui devrait progressivement s'élever à 60 milliards de dollars à l'horizon 2025. Le plan prévoit de structurer une industrie de l'IA d'une valeur de 150 milliards de dollars d'ici 2030, année où la Chine espère constituer le premier centre d'innovation mondial en intelligence artificielle.

5. Guliver's Troubles, or the Setting of American Foreign Policy, New York (N. Y.), McGraw-Hill, 1968.

6. Cf. le résumé du rapport du général Lin Jianchao sur « les défis en matière de prise de décision intelligence lancés par AlphaGo », publié par le site Kunlunce.cn le 18 avril 2016. 
Ce brusque recentrement des intérêts chinois autour de l'IA pourrait paraître énigmatique, n'était la place singulière qu'occupe le jeu de go dans la civilisation chinoise. Ce jeu est né en Chine au premier millénaire avant notre ère. Difficile d'accès, il exige de l'entendement une grande agilité. Il est étroitement lié à la tactique militaire traditionnelle : on en retrouve les principes dans L'Art de la guerre, de Sun $\mathrm{Zi}$, et, depuis les Huns, il s'est imposé comme le loisir de prédilection de la classe militaire et aristocratique. Après la Seconde Guerre mondiale, le nouveau régime communiste en fit même l'étendard du nationalisme chinois, d'autant qu'il fut dominé pendant des décennies par les Japonais, les rivaux historiques ${ }^{7}$. Par conséquent, la victoire d'un programme informatique, conçu qui plus est par Google, symbole par excellence de l' « impérialisme post-moderne américain », est loin d'être anecdotique. Elle eut l'effet d'un choc sur la population et ses dirigeants, traduit avec inquiétude par la presse officielle : «Sommes-nous préparés à l'essor de l'intelligence artificielle ? » ${ }^{8}$.

Désormais, les Chinois et les Américains font la course en tête. Les autres pays demeurent manifestement en retrait, sauf dans des secteurs d'activité très précis, comme l'industrie de défense, où la Corée du Sud, Israël et la Russie, par exemple, ont affiché des ambitions similaires, bien que la réalité de leurs avancées soit difficile à évaluer. Dans le domaine des systèmes d'armes létales autonomes, surnommés « robots tueurs » par leurs détracteurs, la Corée du Sud fait figure de pionnier avec sa sentinelle robotisée SGR-A1, développée au début des années 2000 par Samsung et l'université de Corée. Après avoir dévoilé son premier prototype en 2006, la Corée du Sud aurait commencé à déployer la sentinelle le long de la zone démilitarisée intercoréenne en 2014. Configurée pour accomplir toutes les tâches d'une sentinelle ordinaire, depuis l'identification jusqu'à l'engagement du feu, de façon autonome, elle dispose également d'un système de reconnaissance vocale fondé sur la technique du machine learning ${ }^{9}$.

Toutefois, il est douteux que ces pays puissent rivaliser avec la puissance financière chinoise, ou même avec l'avance technologique des États-Unis : la France prévoit d'investir

7. Élisabeth Papineau, «La culture arrogante du go. Le weiqi, une façon chinoise de voir le monde », Perspectives chinoises, $\mathrm{n}^{\circ}$ 62, 2000, p. 45-56.

8. «Are We Prepared for the Rise of Artificial Intelligence? », China Daily, 11 mars 2016.

9. John E. Pike, « Samsung Techwin SGR-A1 Sentry Guard Robot », GlobalSecurity.org, 7 novembre 2011. 
1,5 milliard d'euros d'ici la fin du mandat d'Emmanuel Macron ; l'Allemagne a annoncé un plan de 3 milliards d'euros étalé jusqu'en 2025. Tous les autres pays ont des prétentions plus modestes. En outre, de l'aveu même d'une responsable du Pentagone, la Chine aurait déjà investi 12 milliards de dollars dans les systèmes d'intelligence artificielle en 2017 : ces investissements devraient même dépasser les 70 milliards de dollars d'ici $2020^{10}$, soit trois fois la somme annoncée par les autorités chinoises. C'est la raison pour laquelle nombre d'États, et les États-Unis au premier chef, entendent exploiter la puissance économique et technologique du secteur privé pour compenser la faiblesse relative des investissements publics.

\section{Vers une compétition transnationale}

Dans les démocraties libérales, le retrait progressif de l'État de la sphère économique au profit du marché a peu à peu ôté aux pouvoirs publics la possibilité de mobiliser à leur guise les ressources du pays pour faire face à de potentiels adversaires. En cas d'affrontement, la dépendance de l'État à l'égard du secteur privé n'en est que plus étroite. À l'inverse, la Chine fonde précisément son essor sur la capacité du pouvoir à entraîner et à orienter l'appareil économique et scientifique national afin d'accomplir son dessein : recouvrer son statut de première puissance.

\section{Le complexe techno-partidaire chinois}

La rivalité sino-américaine pour la précellence en matière d'intelligence artificielle s'articule suivant deux méthodes différentes. En Chine, l'État et, plus précisément, son principe actif, le Parti communiste, établissent les grandes orientations stratégiques du pays et sont les principaux agents de leur mise en œuvre, tandis que les industries technologiques et les entreprises numériques remplissent in fine des fonctions exécutives. Le mode d'actuation militaire de l'IA illustre ce schéma dirigiste : sous la direction de la Commission militaire

10. Interview du lieutenant général VeraLinn Jamieson, chef d'état-major adjoint chargé du renseignement, de la surveillance et de la reconnaissance de l'Us Air Force, in Oriana Pawlyk, « China Leaving Us Behind on Artificial Intelligence: Air Force General », Military.com, 30 juillet 2018. 
centrale, directement dépendante du Parti communiste, la stratégie chinoise consiste à dicter aux sphères économique et scientifique la direction de leurs activités, à les financer, afin qu'elles innovent dans le sens des intérêts de l'Armée populaire de libération. Le but est de réaliser une parfaite « intégration civilo-militaire », selon l'expression de Xi Jinping ${ }^{11}$, fondée sur une convergence de moyens et d'objectifs qu'autorise la nature duale (civile et militaire) des technologies d'intelligence artificielle. Il en résulte la constitution d'un complexe technopartidaire, une structure d'étroites interrelations entre la sphère technologique et numérique et les différents organes infrastructurels du Parti communiste. C'est ainsi qu'en mars 2017 la Commission nationale pour le développement et la réforme a désigné le groupe Baidu pour diriger le Laboratoire national d'ingénierie des technologies d'apprentissage profond, dédié à la recherche dans le domaine du deep learning, de la vision par ordinateur, de l'identification biométrique et des différentes formes d'interaction homme-machine, en partenariat avec l'université Tsinghua, l'université Beihang, l'Institut chinois de recherche en information et communication et l'Académie chinoise des sciences. Tous ces organismes sont structuralement liés au Parti communiste : l'université Beihang, par exemple, coordonne une part majeure de la recherche technologique aérospatiale de l'Armée populaire de libération, notamment ses programmes de développement de drones sous-marins et aériens autonomes, de «guerre en essaim » (swarm warfare) et d'interaction homme-machine. Elle a par ailleurs mis en place le premier programme de mastère en IA de Chine, en partenariat avec des industriels, dont Baidu ${ }^{12}$.

Avant même que l'IA ne fût une priorité nationale, l'Armée populaire de libération avait déjà noué de solides liens avec les entreprises technologiques, à l'instar de la start-up iFlytek (Keda Xunfei), spécialisée dans la reconnaissance vocale, qui reçut dès 2014 une «licence de recherche scientifique et de production d'armes et d'équipements » du Bureau de l'industrie des sciences et technologies de la défense nationale, lui permettant de répondre aux commandes de l'armée ${ }^{13}$. Depuis, l'intégration des grandes entreprises numériques à la

11. «Xi Jinping préside la première séance plénière du Comité central de développement de l'intégration civilo-militaire », Xinhua News Agency, 20 juin 2017.

12. Université Beihang, « Direction de la recherche sur l'intelligence artificielle 2017, double diplôme de mastère. Génie logiciel, Beihang Software College », Présentation, 19 mars 2017, en ligne.

13. Liu Xinglong, «Keda News a obtenu une licence de production d'armes », Sina, 11 avril 2014. 
stratégie d'État s'est approfondie. Le gouvernement a placé chacune d'elles à la tête de programmes nationaux en IA: Baidu aux véhicules autonomes, Alibaba aux smart cities, Tencent à l'imagerie médicale, SenseTime à la vision par ordinateur, et iFlytek à l'intelligence vocale. Cette stratégie semble porter ses fruits : l'entreprise YaTrans a mis ainsi son outil de traduction automatique (Mavericks), basé sur la reconnaissance vocale, à la disposition du ministère de la Sécurité de l'État (en charge du contre-espionnage), des gouvernements provinciaux du Tibet et du Xinjiang, et de la région militaire de Shenyang, à la frontière nord-coréenne ${ }^{14}$. Au total, le complexe techno-partidaire, sur lequel reposent le modèle dirigiste et la stratégie d'intégration civilo-militaire chinois, n'est guère éloigné de la stratégie suivie par les États-Unis pendant la guerre froide.

\section{Les États-Unis, l'IA et les GAFAM}

Les États-Unis disposent aussi d'une puissante industrie technologique et numérique. Avec un chiffre d'affaires de 700 milliards de dollars en 2018, Google (Alphabet), Amazon, Facebook, Apple et Microsoft sont sans réel équivalent dans le monde. Or ils maîtrisent les financements ainsi que la recherche et développement américains - et au-delà - en intelligence artificielle. En 2017, ces entreprises ont dépensé plus de 70 milliards de dollars en recherche et développement.

À rebours du modèle chinois, l'administration américaine entend moins canaliser l'industrie technologique que l'inciter à partager ses innovations, à travers des subventions, des dégrèvements fiscaux et des contrats avantageux. Cependant, il n'existe toujours pas de stratégie de développement déterminée en IA, malgré la succession de «plans » depuis le dernier mandat de Barack Obama ${ }^{15}$. Ces différentes initiatives, y compris l'Executive Order présidentiel et l'AI Strategy du département de la Défense, dévoilés en février $2019^{16}$,

14. «Partners », YaTrans.com, $1^{\mathrm{er}}$ mars 2019.

15. Cf., par exemple, National Science and Technology Council, The National Artificial Intelligence Research and Development Strategic Plan, NITRD.gov, octobre 2016.

16. Executive Order on Maintening American Leadership in Artificial Intelligence, WhiteHouse.gov, 11 février 2019 ; Summary of the 2018 Department of Defense Artificial Intelligence Strategy: Harnessing AI to Advance Our Security and Prosperity, Media.Defense.org, 12 février 2019. 
reprennent in globo les grandes lignes de la Third Offset Strategy de 2014, soit le recours à des mécanismes incitatifs en direction du secteur privé, afin de l'encourager à innover et à transférer ses technologies à l'administration américaine et, au premier chef, au département de la Défense.

Toutefois, les grandes firmes technologiques et numériques sont loin de se cantonner à un simple rôle d'exécutant. Bien plus, elles participent activement à la définition de cette antistratégie qui leur est profitable - même de façon formelle, au sein de la Commission de sécurité nationale pour l'intelligence artificielle, dont l'institution fut décidée par le Congrès, en mai $2018^{17}$. Parmi ses membres se distinguent les PDG d'Amazon Web Service et d'Oracle, les directeurs de Microsoft Research Labs et de Google Cloud AI, et surtout son président, Eric Schmidt, l'ancien PDG de Google et d'Alphabet.

La position de ce dernier, au carrefour des secteurs privé et public, est paradigmatique du rôle que les entreprises numériques tiennent dans la définition de la stratégie américaine en matière d'intelligence artificielle. Pendant la campagne présidentielle de 2008, Eric Schmidt soutint financièrement la candidature de Barack Obama, dont il fut le conseiller privé avant d'occuper plusieurs postes officiels à la Maison-Blanche. En mars 2016, le secrétaire à la Défense, Ash Carter, le nomma président du Defense Innovation Board, poste qu'il occupe toujours. Au sein de cet organisme chargé de conseiller le département de la Défense en matière d'innovation organisationnelle et d'acquisition technologique, avec une nette priorité accordée à l'IA, Eric Schmidt vitupère régulièrement contre les pesanteurs bureaucratiques du Pentagone, prêche sa réforme complète et sa mise en retrait au profit du secteur privé ${ }^{18}$, tout en conservant ses fonctions de conseiller technique et de membre du conseil d'administration d'Alphabet. D'ailleurs, certains des grands contrats liant le Pentagone et Google-Alphabet furent passés lorsqu'il cumulait la direction de l'entreprise et celle du Defense Innovation Board. C'est le cas du projet Maven, lancé en avril 2017 par le secrétaire adjoint à la Défense, Robert Work, par lequel Google fut chargé de concevoir des systèmes de reconnaissance d'images, basés sur la technique du deep learning, pour renforcer les capacités de surveillance

17. National Defense Authorization Act for Fiscal Year 2019, section 1051, « National Security Commission on Artificial Intelligence ».

18. «Statement of Dr. Eric Schmidt, House Armed Services Committee », Docs.House.gov, 17 avril 2018. 
et d'identification de cibles par les drones de l'armée et du renseignement américains. Démis de ses fonctions en juillet 2017, quelques mois après l'arrivée au pouvoir de Donald Trump, Robert Work est aujourd'hui l'adjoint d'Eric Schmidt à la tête de la Commission de sécurité nationale pour l'intelligence artificielle.

Loin d'être un cas isolé, le parcours ondoyant d'Eric Schmidt est symptomatique de la place qu'occupent les firmes transnationales numériques au sein de l'État. D'autres personnalités « duales », venues de Microsoft, d'Amazon, de Facebook ou d'Oracle, décrivent des trajectoires analogues, à l'instar de Peter Thiel, président-fondateur de Palantir et ancien conseiller du président Trump. Au-delà des agissements individuels, ces entreprises ont établi une stratégie d'influence, faite de lobbying et d'entrisme, qui semble porter ses fruits, eu égard aux avantages financiers et juridiques que l'État leur accorde. En 2018, les GAFAM, au sens strict, ont dépensé plus de 64 millions de dollars en lobbying. Durant la session parlementaire 2017-2018, trois cent soixante-quatre des quatre cent cinquante lobbyistes de ces firmes $(81 \%)$ occupaient auparavant au moins un poste au sein des institutions (MaisonBlanche, Congrès, départements ou agences) ${ }^{19}$.

Surtout, l'expansion internationale de leurs activités ne semble rencontrer aucune restriction de la part des pouvoirs publics américains, quand bien même pourrait-elle s'opposer aux intérêts de l'État. Lorsque Google ouvrit son centre de recherche et de formation en intelligence artificielle à Pékin, en décembre 2017, les relations sino-américaines s'étaient profondément détériorées, en particulier parce que Washington craignait déjà de se faire ravir sa supériorité technologique et in fine son statut d'hegemon, par suite des succès chinois en IA. Cela n'a guère freiné les ambitions de l'entreprise, ni celles d'Amazon et de Microsoft, qui chacune annonça l'ouverture de structures similaires à Shanghai, lors de la World Artificial Intelligence Conference, en septembre 2018, une manifestation organisée par plusieurs organes étatiques chinois, dont la Commission nationale du développement et de la réforme, la grande agence de planification économique d'État.

Ces entreprises s'instituent de fait en acteurs des relations internationales. Par-delà leur prépondérance et leur rôle diplomatique dans le cyberespace ${ }^{20}$, elles maîtrisent des

19. «Influence \& Lobbying », OpenSecrets.org.

20. Microsoft appelle à une régulation du cyberespace. 
infrastructures essentielles partout dans le monde, telles que des câbles sous-marins transcontinentaux de fibre optique et des centres de données, nécessaires au fonctionnement de leurs systèmes d'intelligence artificielle et, plus généralement, au Web. En outre, elles tendent à placer certains États dans leur dépendance, notamment les alliés des États-Unis qui désirent convertir leur économie à ces nouvelles technologies. À lui seul, Google a inauguré une douzaine de centres de recherche en IA hors des États-Unis, en Europe, au Canada, en Asie orientale, au Moyen-Orient et en Afrique; il a été également présenté comme un partenaire privilégié de plusieurs stratégies étatiques en IA, dont celle de la France, à travers sa filiale britannique. En Italie, Diego Piacentini, un ancien cadre dirigeant d'Apple et d'Amazon, a même été chargé de conduire la politique numérique du gouvernement et de le représenter dans ce domaine à l'étranger, lors de G7 ou de G20 entre 2016 et 2018. Le rôle majeur qu'accordent les États à l'intelligence artificielle et la place centrale qu'occupent ces firmes dans ce nouvel environnement technologique induit naturellement une transnationalisation des relations internationales. Celle-ci se nourrit de la conviction néolibérale, largement partagée à la tête des démocraties occidentales, d'un nécessaire effacement de l'État dans la plupart des activités économiques, au bénéfice d'entreprises qui véhiculent ce type de discours. Ou, autrement dit, d'un rééquilibrage manifeste de la politique internationale, d'une dissémination de la puissance, au profit des grandes firmes numériques. 